Voix et Images

voixetimages

\title{
Vers la découverte de l'identité : les trois premiers romans de Gilbert Larocque
}

\section{Els Post-Pieterse}

Volume 3, numéro 2, décembre 1977

Victor-Lévy Beaulieu

URI : https://id.erudit.org/iderudit/200107ar

DOI : https://doi.org/10.7202/200107ar

Aller au sommaire du numéro

Éditeur(s)

Les Presses de l'Université du Québec

ISSN

0318-9201 (imprimé)

1705-933X (numérique)

Découvrir la revue

Citer cet article

Post-Pieterse, E. (1977). Vers la découverte de l'identité : les trois premiers romans de Gilbert Larocque. Voix et Images, 3(2), 277-301.

https://doi.org/10.7202/200107ar d'utilisation que vous pouvez consulter en ligne.

https://apropos.erudit.org/fr/usagers/politique-dutilisation/ 


\section{Vers la découverte de l'identité: \\ les trois premiers romans de Gilbert Larocque}

Avant la publication récente de Serge d'entre les morts ${ }^{1}$, Gilbert Larocque avait publié trois romans: le Nombri/2, Corridors ${ }^{3}$, et Apres la boue ${ }^{4}$. II y a une liaison étroite entre ces trois premiers livres. Les protagonistes de ces romans sont, chacun à sa manière, à la recherche de leur identité. Et le chemin qui conduit au moi perdu est obscurci par des frustrations qui prennent leur origine dans l'enfance. La constellation des complexes s'éclaircit toutefois dans Après la boue. A partir d'une analyse du caractère et de la vie de l'héroïne de ce roman, une étude diachronique des trois livres permet de voir un progrès dans les efforts des héros pour retrouver leur identité. En lisant et surtout en relisant les romans de Larocque je fus frappée par les variations pronominales au point de me demander si une étude des pronoms personnels pouvait contribuer à notre compréhension de l'œuvre. L'élaboration de cette idée m'a menée à croire que le rôle des pronoms personnels qui, à ma connaissance, n'est jamais exploré dans la littérature québécoise, peut être très significatif surtout dans des romans où, comme dans ceux de Larocque, il y a un emploi varié des pronoms personnels.

\section{Après la boue}

Après la boue est l'histoire de Gabrielle racontée par elle-même. Celleci est donc en même temps l'héroïne et la narratrice: «je ressentais le besoin de raconter mon histoire il fallait que je m'en délivre il fallait que j'en accouche et parler au docteur Narcix ne me suffisait plus et j'accrochais d'autres malades et je racontais l'histoire de Gabrielle..." (p. 205) La narratrice s'est d'abord adressée "au docteur Narcix". Ce nom évoque le mot Narcisse et ainsi it pourrait indiquer qu'elle s'est adressée à ellemême. Faute d'être satisfaite par Narcix elle s'adresse ensuite "aux autres malades". Dans un sens un peu large on pourrait dire que "les autres malades" sont les autres êtres humains y compris les lecteurs d'Après la boue.

Je crois qu'il y a trois passages au temps de l'écriture dans lesquels la narratrice s'adresse directement au lecteur pour commenter la technique de narration (p. 14, 15, 18, 22, 23). Abstraction faite de ces interven- 
tions le livre se divise en quatre parties dont la première se situe au temps de la préaventure et les trois autres au temps de l'aventure:

1. les souvenirs d'enfance (p. 7-18)

2. résumé de l'histoire proprement dite (p. 18-22)

3. I'histoire proprement dite (p. 23-200)

4. la postface (p. 200-208)

L'histoire proprement dite se subdivise en trois parties qui toutes les trois finissent par une crise:

1. la crise autour de Roch (p. 23-81)

2. la crise autour de Fœtus (p. 82-142)

3. la crise autour du pere (p. 143-200)

Cette histoire qui décrit une année de sa vie raconte l'effort de Gabrielle pour trouver son identité. La postface présente le résultat de cette recherche difficile et pénible.

Dans la première partie (p. 7-18), Gabrielle raconte quelques-uns de ses plus anciens souvenirs. Ces expériences de la petite Gabrielle forment la base de ses complexes. "C'était samedi, un peu après la mort de grandpère...» (p. 156) «... il ne resta plus dans la chambre que la noirceur et elle-même l'enfant Gabrielle avec ses terreurs folles... Gaby attendait le sommeil... elle ne dormait pas... " (p. 7) Voilà notre première rencontre avec Gabrielle. Elle a cinq ans (p. 58), elle se trouve dans sa chambre noire et close et elle a peur: "oui moi j'avais toujours peur" (p. 7). D'où vient cette peur? Gabrielle ne raconte qu'un souvenir antérieur à ce samedi: "... je me rappelle bien, un soir que je me déshabillais un visage blême était apparu là dans ma fenêtre c'était un homme c'était un rôdeur de nuit noire..." (p. 8) Effrayée Gabrielle appelle sa mère afin d'être protégée: “moman il y a un homme» (p. 97). Mais c'est son père, le grand protecteur de la famille, "un mari c'est supposé défendre sa femme, ah!" (p. 11), qui accourt "tout pâlot tout grouillant nerveux" (p. 8). «... il avait entreouvert (presque pas) la porte de la cuisine et avait regardé dehors et il avait dit c'est rien y a personne c'est rien que son imagination mais il avait sa main et.sa gueule qui tremblotaient..." (p. 8) Alors Gabrielle se rend compte que son père lui-même a peur et que pour masquer sa peur il prétend ne pas la croire. Elle se sent dépourvue de protection, “livrée sans défense à sa propre peur, compliquée de l'angoisse que fait naître en [elle] l'écroulement de ses dieux...5” (B, p. 244)

II y a donc une double angoisse. La désillusion qu'elle éprouve au sujet de son père fait naître en même temps des sentiments de haine et d'agressivité envers lui, sentiments qui se confondent avec l'agressivité qui se forme en défense contre le danger extérieur: le guenillou. Tout ça devient encore plus complexe quand on se rend compte que la conscience morale réprouve tout sentiment négatif à l'égard des parents. Les sentiments de Gaby envers son père la rendent coupable et cette culpabilité intensifie son angoisse. Puisque cette angoisse est insupportable elle provoque des mécanismes de défense. D'un côté les sentiments négatifs à l'égard du père sont refoulés et de l'autre côté l'angoisse se fixe sur un objet. Cet objet s'impose logiquement à Gaby: le guenillou. (B. p. 241) 
Le refoulement des sentiments crée une «ombre ${ }^{6}$ » «qui est une sorte de 'double' incarnant le refusé, le non-vécu, le refoulé et qui en certaines circonstances réclame avec insistance satisfaction" $(B, p .77)$. Cette ombre est une chose effrayante qui anime et peuple l'ombre réelle, le noir, la nuit. (B, p. 253-254) Ceci explique les «terreurs folles" (p. 7) de Gaby dans sa chambre noire. Pour compliquer les choses la chambre de Gabrielle est hantée par la tante Éva (p. 11): “... ce masque de morte... Éva la folle qui exhumait à tout propos ses photos de jeunesse où j'apercevais un visage qui ressemblait trop au mien. » (p. 12) Par cette ressemblance l'ombre de Gabrielle adopte le visage de la tante Éva. Quand Gabrielle parle d'une “... présence formidable, [...] plus noire que la nuit, une ombre de cauchemar qui pulsait dans toute la chambre comme un ccur monstrueux..." (p. 7), il s'agit donc de trois ombres superposées: son ombre, l'ombre de la tante Éva, et l'ombre réelle, le noir. Le double de Gaby incorpore, outre les sentiments inadmissibles à l'égard de son père, la mort et la folie de la tante Éva. Quand le double "demande satisfaction» cela implique une menace de folie et de mort pour Gabrielle. (Cf. p. 149 et 156.)

Son angoisse qui dès le refoulement est devenue vague et incompréhensible, s'est fixée sur le guenillou. Pour Gabrielle le guenillou est "un rôdeur... un maniaque avec des couteaux et des rasoirs... " (p. 97) avec lesquels «il lui ouvre lentement le ventre et la déchire» (p. 8) et finalement: “il lui mâche les tripes il lui croque son cceur il suce tout son sang il la dévore telle quelle! grouf !..." (p. 8). Par l'intervention de l'oncle Émilien la peur du guenillou se transforme en une obsession sexuelle. L'oncle Émilien veut violer sa nièce «il est rouge-viol" (p. 16). Gaby est paralysée par la peur: “... elle n'ose pas parler et encore moins bouger car son oncle est fort, il est brutal et quand il est ivre il se bat, il fait saigner les gens... et surtout il a dans sa poche un gros couteau à deux lames avec un tire-bouchon et ouvre-bouteille et un pic à glace..." (p. 16) Gaby fait elle-même l'association entre Émilien et le guenillou: «il n'a quand même pas dit je vais t'ouvrir le ventre et t'arracher toutes les tripes si tu cries ou si tu grouilles d'un poil, mais il a des yeux qui disent beaucoup plus". (p. 16) La nature des images indique que, pour Gaby, Émilien est la personnification du guenillou. Cette première expérience si malheureuse influencera sa vie sexuelle. Pour elle l'amour charnel sera viol et l'homme qui commettra ce «viol» sera un guenillou.

Gabrielle sort de l'enfance avec une frustration complexe. A la base se trouve l'angoisse causée par son père qui a engendré des sentiments de haine et d'agressivité envers celui-ci. A leur tour ces sentiments ont donné à Gabrielle l'impression qu'elle est coupable envers son père. Tous ces sentiments ont été refoulés et ont donné naissance à un double. Ce double a pris le visage de la tante Éva qui y a ajouté la menace de la mort et de la folie. L'angoisse qui continue à se faire sentir s'est fixée sur le guenillou, une fixation qui par l'intermédiaire de l'oncle Émilien a abouti à une obsession sexuelle. La frustration peut donc être divisée en trois complexes: 
1. la haine coupable pour le père

2. la hantise du double

3. l'obsession sexuelle.

A plusieurs reprises la voix de la mère intervient dans les souvenirs de Gaby. La mère semble représenter une force plus positive. Ses paroles sont des ordres, mais des ordres clairs. Cette clarté est l'opposé de la confusion dåns laquelle le père laisse Gaby. Les mots de la mère sont loi pour Gabrielle et elle sait que violer la loi c'est risquer une punition. Sa mère lui a défendu de salir sa «belle robe blanche» (p. 8) et de «se caresser devant son miroir» (p. 15): «c'est péché! sa mère le lui défend il faut le dire à confesse il faut se repentir vrai JE ME PROPOSE MOYENNANT VOTRE SAINTE GRÃCE DE NE PLUS oui il faut se repentir tu irais droit en enfer petite vicieuse !...» (p. 16) II semble que l'énergie de la mère s'oppose surtout aux poussées instinctives et qu'elle adopte sur ce point les idées de l'Église. Gabrielle voit sa mère comme "un archange" (p. 10): angélique et pure mais en même temps supérieure. Sa mère a aussi un rôle protecteur. Quand Gaby se sent menacée par le guenillou elle appelle sa mère (p. 97). Abandonnée par sa mère, Gaby est livrée aux forces noires du guenillou: "aussitôt que sa mère eut éteint la lumière et fermé la porte... il ne resta plus dans la chambre que la noirceur et elle-même l'enfant Gabrielle avec ses terreurs folles..." (p. 7) La mère est cruelle: elle menace sa petite fille avec le guenillou tout comme l'Église menace avec l'Enfer (p. 16). Elle met Gaby dans une situation sans issue: elle a "fermé la porte" (p. 7). Gaby doit dormir: "t'es mieux de dormir de pas dire un mot parce que tu vas voir ça que le guenillou va venir te poigner" (p. 7.) Il semble impossible d'éviter le guenillou. Par peur du guenillou Gaby ne dort pas, mais si elle ne dort pas le guenillou va venir la poigner. L'ordre "de pas dire un mot" exclut même la possibilité de demander la protection de ses parents. Gabrielle est toute seule. Je crois que ces interventions maternelles qui disent à Gaby ce qu'il faut et ce qu'il ne faut pas faire forment la base du surmoi de Gaby.

La personnalité de Gabrielle telle qu'elle s'est formée pendant son enfance présente donc trois facettes:

1. "le moi ordinaire et pour ainsi dire central» $(B$, p. 77$)$

2. une ombre ou un double qui représente "le refusé, le non-vécu, le refoulé ' $(B, p .77)$

3. un surmoi, "un complexe appelé à s'opposer aux autres complexes, à exercer sur eux une régulation » $(B$, p. 129).

L'histoire proprement dite présente la vie de Gabrielle pendant l'année si importante où elle essaie de se regarder en face et finit par se perdre complètement. Se regarder en face veut dire s'accepter telle quelle et si nécessaire «poser des actes importants" ( $p$. 175) pour atteindre ce but. Gaby a trois complexes et au cours de cette année elle va s'en rendre compte et puis s'en affranchir. Le livre est construit autour de trois crises majeures dont chacune réalise l'élimination d'un complexe. Avant l'éclatement d'une crise, il y a une période pendant laquelle Gaby pressent la crise ("elle sut que tout allait de travers" (p. 40)). Chaque crise majeure est précédée d'une crise mineure: 
1. la scène au dancing le Tréponème (p. 60-65)

2. la visite au docteur Médullaire (p. 113-122)

3 . la conversation avec Nathalie et surtout le retour à la maison (p. 177180).

Pendant une crise mineure rien n'est accompli, dans le sens où Gaby n'élimine aucun complexe, mais les symptômes physiques et psychiques sont identiques à ceux d'une crise majeure. Quels sont ces symptômes? Physiquement il y a surtout deux symptômes: le mal de tête et la sensation d'avoir chaud ou froid ou les deux en même temps. Psychiquement la crise est surtout préparée par des souvenirs d'enfance et par une lutte intérieure entre les différents «moi " de Gaby. Parfois un autre personnage prend la parole pour un de ces moi. Les crises mineures sont dans les trois cas une réaction contre l'intervention d'un autre. Dans le premier cas c'est Roch qui prend la parole pour le surmoi de Gabrielle qui lui défend de quitter son mari : “ta place est à la maison» (p. 56). Dans le deuxième cas c'est le docteur Médullaire qui verbalise le désir de Gaby de tuer fœus: “si je comprends bien vous voudriez avorter?" (p. 119) Dans le troisième cas Nathalie admet la nécessité de régler ses comptes avec son père: "Parce que si on réalise rien, si on pose pas des actes importants ça vaut pas la peine de rêver... hein ?... ni même de vivre. » (p. 175)

Sous l'influence des souvenirs et des interventions extérieures la lutte intérieure s'intensifie et finit par la victoire d'un des moi qui à ce moment impose sa volonté, et fait agir Gabrielle. Les maux de tête et les sensations de chaleur et de froid reflètent ce conflit intérieur. La première crise a lieu le soir du 28 août. Gabrielle quitte son mari Roch et retourne à la maison paternelle. La crise est la lutte finale entre le surmoi de Gabrielle qui défend le mariage comme une institution sacrée assurant une vie tranquille: «j'ai un bon mari, la sécurité, la bonne petite vie tranquille c'est parfait je suis heureuse... » (p. 39) et le double de Gabrielle pour qui le mariage est "une éccurante comédie» (p. 77) dominée par "la loi de la nature [qui pousse les humains à] s'accoupler» (p. 36). Pour Gabrielle le mariage est sécurité d'un côté et accouplement de l'autre. II semble que tous les autres aspects du mariage manquent. A en croire Gaby il n'y a aucune communication spirituelle: “... ils ne parlaient pas, ils n'avaient rien à se dire..." ( $p .45)$, ou émotionnelle: «Elle l'embrassa du bout des lèvres, rite matinal qui ne signifiait rien ou qui signifiait terriblement trop en n'ayant pas de sens..." (p. 46). Elle considère Roch comme une brute, un animal qui satisfait ses besoins naturels et en est content. II mange beaucoup (p. 32) et il aime manger: "peut bien manger, jouisseur..." (p. 68) "Puis dormir comme il dort... son sommeil sacré ses neuf ou dix heures par nuit..." (p. 68). Même ses séances à la selle ne sont pas dépourvues de plaisir: "oui couve sa marde pendant des quinze ou vingt minutes..." (p. 73) Ayant cette image de Roch on peut penser comme Gaby: «puis évidemment sa petite décharge presque tous les soirs" (p. 68). Cette "petite décharge " qui se fait pendant l'accouplement (la terminologie l'indique déjà) est elle aussi une activité bestiale où l'amour ne joue aucun rôle. Gaby compare les rapports sexuels à l'accouplement des chats et des chiens: «... oui 
mais les chiens et les chats, matou saillant, accouplement furieux... (p. 36) “... chien grimpant en extase la chienne..." (p. 77) Avec une idée pareille de la sexualité il n'est pas étonnant que Gabrielle la trouve dégoûtante. II n'y a qu'à lire sa description de la nuit de noces (p. 33-37) pour savoir à quel point "[ça] lui faisait l'effet d'un quartier de viande pourrissant avec odeurs derrière un buisson de dépotoir, quelque chose comme une charogne innommable vautrée sous ce tapis de fleurs fanées..." (p. 34)

Gaby se déclare "obsédée sexuelle" (p. 64) et le lecteur admet sans peine ce diagnostic. Reste la question de savoir comment Gaby en est arrivée là. Heureusement l'auteur fournit gentiment la réponse à cette question en initiant le lecteur aux souvenirs d'enfance de Gaby. Avant d'aborder l'histoire du 28 août il a mis le lecteur au courant de l'existence de l'effrayant «guenillou » qui «... la jette comme un quartier de viande dans sa charrette... [pour lui ouvrir]... lentement le ventre avec un rasoir rouillé... [afin de la dévorer]... vive... " (p. 8) L'auteur lui a aussi appris que plus tard dans la vie de Gaby le guenillou est devenu réalité sous la forme de son oncle Émilien. Pour Gabrielle, Roch est un autre guenillou. Pendant sa nuit de noces elle s'en rend compte: «c'était donc ça faire l'amour! et oui elle l'avait toujours su, oui elle l'avait toujours obscurément pressenti, ce que cela pouvait être..." (p. 33, 34) Les activités de Roch ressemblent à celles du guenillou (p. 8): “... sauvage [il] l'enfile à bout portant, la déchire, l'enfonce, l'écartèle... vous dépeçant en quatre blocs de viande saignante..." (p. 37) Gaby ne peut pas échapper à ce nouveau guenillou parce que Roch «y a droit... on n'achète pas la sécurité du mariage avec rien, payer mon écot, et alors faire son possible... lui donner tout son plaisir... et puis je l'aime après tout, non? puisque je l'ai épousé ça prouve tout, il faut !...s (p. 36)

Pendant la nuit de noces le surmoi qui fait retentir la voix de sa mère: «tu feras ça comme y faut, tu me feras pas honte» (p. 36) est l'aspect le plus fort de la personnalité de Gaby. Le surmoi retiendra cette position dominante pour «cinq ans» (p. 111). Ce ne sera que ce jour d'août où la chaleur est étouffante que le double de Gabrielle l'emportera. Le 28 août Gaby est presque sûre d'être enceinte. L'intervention de cet élément, concret, même palpable, ravive la dispute intérieure en fournissant un nouvel argument: "VOUS VOUS PERPÉTUEREZ A TRAVERS VOS ENFANTS VOUS VIVREZ EN EUX MÊME APRÈS VOTRE MORT ET N'OUBLIEZ PAS QUE LES ENFANTS CONSOLIDENT L'AMOUR DU COUPLE... mais non je ne veux pas me perpétuer non je ne veux pas me transmettre je veux que tout finisse à moi non ça ne changerait rien entre Roch et moi... absurde mensonge! non je ne veux plus mentir c'est assez! MAIS VOUS NE COMPRENEZ PAS QUE ROCH JE LE DÉTESTE!... non ce n'est pas ce que je voulais dire je le jure... non je ne veux pas j'aime mon mari j'aime mon mari j'aime mon mari !...» (p. 63)

C'est là un de ses derniers mensonges à l'égard de Roch. Ce jour même Gaby mettra fin à «l'écœurante comédie» (p. 77). Une fois pour toutes elle se décidera: «c'est assez! non! je ne suis pas sa putain non !... (p. 77) et elle va définitivement quitter Roch. 
La deuxième crise a lieu un dimanche d'octobre. Gabrielle se fait avorter dans le bain et tue ainsi Fœtus. Cet acte dramatique est la fin de la lutte entre la Gabrielle qui défend les joies de la maternité: "la maternité... être mère, penses-y... rien de plus beau, rien de plus noble... " ( $p$. 126 ) et l'autre Gabrielle qui "déteste fœtus " et qui veut "tuer fœtus " ( $p$. 120). Le conflit intérieur autour de fœtus avait déjà commencé le 28 août: "et si vraiment fœetus nageant dedans... ah c'est terrible non je sens que je ne pourrais pas non je ne veux pas!... voyons! Gaby! raisonne-toi un peu... joies de la maternité..." (p. 69) Une partie de Gaby refuse fœtus non pour des raisons pratiques ou économiques, "la question n'est pas là" (p. 69), mais parce qu'elle a peur: "oh j'ai peur de quelque chose j'ai peur et si c'était vrai que mon ventre? » (p. 69)

D'où vient la peur de Gaby? Regardons l'image que Gaby a des couches: «... ton utérus en contractions son col dilaté ton sexe distendu par sa grosse tête molle... et poussez fort... on dirait que je vais éclater oh forcer encore et encore un peu et voilà ça y est ça sort de moi c'est rougeâtre gélatineux gluant c'est mon fils c'est ma fille ô bonheur ${ }^{7}$ ! " (p. 69, 70) Cette image de la naissance rappelle l'accouplement avec Roch qui force aussi Gaby à «distendre le sexe» ( $p$. 77): «j'ai la fourrante serrée comme un anus mais il est fou il veut que je m'écartèle encore plus" (p. 77) et la mort du grand-père de qui sort aussi une « substance rouge oh très rouge et grumelée glaireuse sanglante pituite qui bougeait clapotait..." (p. 58). La naissance liera Fœtus à “l'accouplement et par là au complexe sexuel de Gaby. La naissance ressemble dans la fantaisie de Gaby à la mort du grand-père. Fœtus symbolise donc en même temps la naissance et la mort. II y a encore un autre aspect à Fœtus, il est une sorte de double de Gaby: "VOUS VOUS PERPÉTUEREZ A TRAVERS VOS ENFANTS VOUS VIVREZ EN EUX MÉME APRÈS VOTRE MORT » (p. 63). Fœtus est l'incarnation du double à l'intérieur d'elle-même. Par le truchement de Fœtus elle pourrait rejeter son double. En portant Fœtus, par contre, Gaby pressent qu'elle pourrait elle-même se transformer en son double. Elle fantasme que, femme enceinte, elle ressemblera à la tante Éva, son ombre qui l'a hantée depuis son enfance: “... pourvu que je n'aie pas le masque de grossesse! ça c'est laid gueule de lépreuse, museau vérolée, l'horreur du quartier, faire peur aux enfants..." (p. 126) Ce portrait rappelle la tante "Éva ce masque de morte avec sa gueule flétrie" (p. 12) qui était l'horreur du quartier (p. 158) et faisait peur aux enfants, notamment à l'enfant Gabrielle.

Pour résumer on peut constater que Fœtus est lié à tous les complexes de Gaby: comme produit de l'amour charnel et par la naissance il rappelle le complexe sexuel; comme futur enfant il renforce la hantise du double. La haine pour son père qui est le complexe primaire de Gaby est refoulée dans le double. Symbole du double, Fotus comporte donc cette haine. Ceci explique les pulsions agressives envers son père (p. $87,88,96$, 135). Dans cette partie il y a beaucoup de souvenirs dans lesquels le père joue un rôle principal. Ils semblent suggérer que la liaison entre le père 
et Fœtus est encore plus étroite. Cette série de souvenirs ( $p$. 97-106) commence par une émergence du plus ancien souvenir: «un visage grimaçant... à la fenêtre de ma chambre il y a un voyeur il y a un rôdeur dans la cour... » (p. 8).

La réaction inadéquate de son père provoque chez Gaby une profonde angoisse à l'égard du guenillou. Cette angoisse provient d'une cause plus constante: la perte de l'appui paternel. C'est dans l'angoisse qu'on trouve les racines du dédain et de l'agressivité que Gaby ressent à l'égard de son père. Les souvenirs suivants racontent l'évolution de ses sentiments à l'égard de son père. Le premier, suivant la chronologie, peint la petite Gabrielle qui dans un mouvement presque inconscient «projette hors d'elle” (p. 103) la haine et l'agressivité qu'elle ressent pour son père: “... l'enfant Gabrielle avait écrit soigneusement sur une petite feuille de papier rose 'je voudrais que tu sois mort',... cette petite phrase devenait un témoin gênant de quelque chose qu'elle était encore incapable d'exactement identifier...» (p. 103) Malgré tous les efforts de Gaby pour annihiler ses mots: elle jette loin d'elle le crayon, elle déchire le papier puis mange les morceaux, «rien n'avait été détruit pour autant» (p. 104). Finalement elle fait un acte expiatoire, espérant neutraliser ainsi la signification des mots. Avec une goutte de sang elle écrit sur un papier rose: “je t'aime, je ne veux pas que tu meures" (p. 106) et elle avale le morceau de papier. En avalant ces deux morceaux on pourrait dire qu'elle assume une double personnalité, qu'elle établit les fondements pour les luttes intérieures à venir. II est évident qu'il y a un parallèle entre ce souvenir et la situation actuelle. Le dilemme d'autrefois se répète: “je veux tuer fœtus» d'un côté et "je m'en vais t'aimer" de l'autre. Au milieu de la crise dans le cabinet du docteur Médullaire (p. 120) ce parallélisme est explicité. Quand Gaby a perdu le contrôle d'elle-même, "elle avait l'impression que les mots entraînaient avec eux sa pensée, cette pensée secrète qu'elle avait constamment refoulée au fond d'elle... JE VOUDRAIS QUE TU SOIS MORT! » (p. 119-120)

La haine et l'agressivité pour son père se projettent sur Fœtus. Mentalement Gaby fait une association entre son père et fœtus. Est-ce que cette association est justifiée? Avant de répondre à cette question il faut d'abord compléter l'image que Gaby se fait de son père. Elle raconte deux autres souvenirs à son sujet: «... autrefois, devant chez nous, il y avait un arbre... puis un jour ils ont coupé l'arbre... ». (p. 85). Pour Gabrielle «c'était triste» ( $p$. 98) mais son père réagit tout autrement: «... mon père n'était pas encore aveugle ce temps-là, dans la fenêtre du salon sa face contente et grimaçant hilare de voir l'arbre assassiné, jouissant du spectacle, pensez! l'arbre ne menacerait plus sa coquille délabrée où il s'était incrusté... $(p$ ( 85). Incrusté dans sa coquille le père est impuissant tout comme un fcetus "incrusté" (p. 19) dans le ventre de sa mère. L'arbre dans son sens symbolique rappelle au père sa virilité et sa responsabilité comme chef de famille. Comme il n'a jamais pu ou voulu assumer ce rôle, il est donc très compréhensible qu'il soit heureux de voir disparaître ce symbole gênant. C'est 
avec enthousiasme qu'il encourage les ouvriers: “sacrez-moi ça à terre» (p. 98). La coupe de l'arbre à la fois dans son sens réel et dans son sens symbolique ne rend les sentiments de haine et d'agressivité de Gaby que plus vivants.

D'un côté les émotions négatives envers son père et de l'autre côté la notion que de tels sentiments ne sont pas admissibles, qu'il faut aimer son père, voilà la situation psychique ambivalente dans laquelle Gaby se trouve quand à l'âge de quinze ans elle apprend que son père a eu un accident grave (p. 99). Cet accident, qui provoque la cécité du père, ressuscite «cet étrange sentiment noir, cette oppression insoutenable (p. 101). "Au fond d'elle" (p. 101) sont les mots qu'elle a écrits: "je voudrais que tu sois mort" (p. 103). Maintenant il semble que sa demande a été exaucée et elle se sent coupable et angoissée: “... elle était terrifiée de quelque chose mais de rien..." (p. 100). Elle s'imagine plusieurs causes de mort parmi lesquelles surtout la dernière me semble significative: "... quel héros pensez! il a voulu sauver cette jeune fille qu'ils essayent de violer... " (p. 100). Ceci me semble une image compensatrice pour Gaby qui aurait aimé avoir un père héroïque, protecteur des petites filles. Elle sait pourtant trop bien que son père a réagi comme un lâche quand son frère Émilien a essayé de violer sa fille à lui, Gabrielle. Quand Gaby apprend que son père n'est pas mort mais 'seulement' aveugle «... elle a... honte de se sentir soulagée” (p. 102). Soulagée parce qu'elle ne se sent plus coupable, honteuse parce qu'elle n'est pas triste "c'était doux et bienfaisant en elle. L'image finale du père de Gaby est donc celle d'un impuissant (l'arbre est coupé), "incrusté dans sa coquille» (p. 85) et aveugle, et par conséquent incapable de se débrouiller sans aide. II ne sait ni se défendre (p. 134, 135, 136) ni se nourrir proprement (p. 131, 134). Je crois que cette image justifie suffisamment l'association entre lui et Fœtus. Gabrielle veut tuer Fœtus non seulement parce qu'elle veut faire disparaître tout souvenir de Roch mais encore parce qu'elle espère que de cette façon elle peut exorciser sa haine et son agressivité envers son père. Dans la troisième partie d'Après la boue on verra que cet espoir est faux.

La troisième crise a lieu au mois de mai. Gabrielle bat son père, quitte la maison et finit par perdre tout contrôle d'elle-même. Déclarée folle elle est internée dans une institution psychiatrique. Cette crise constitue la fin de la lutte intérieure qui se trouve à l'origine de tous les autres conflits. D'un côté il y a Gabrielle qui hait son père: "je voudrais que tu sois mort " (p. 103) et de l'autre côté Gabrielle qui veut aimer son père: “je t'aime je ne veux pas que tu meures" (p. 106).

Dès l'origine de ce conflit Gaby a refoulé ses sentiments négatifs envers son père. Le double qui s'est alors formé a pris le visage de la tante Éva. L'association entre le double et la tante Éva a fait du double une menace de mort et de folie. En tuant Fœtus, Gaby a éliminé son double. Par conséquent toutes les émotions qui y ont été refoulées sont maintenant à découvert. Gaby est forcée de faire face à ces émotions. Elle doit les accepter comme une facette authentique d'elle-même et elle sent le 
besoin d'agir en conséquence. Ceci explique son agressivité envers son père, un des sentiments jusque-là bien gardés par le double. Ce jour de mai en regardant son père, “Gaby se sentit dans les mains un lancinant besoin de griffer et de frapper sec et d'écraser... des boues noires se détachaient et remontaient lentement du fond d'elle et de son passé... » (p. 154). II faut sa cousine Nathalie "ma sœur le beau moi-même" (p. 176) pour faire comprendre à Gaby la vraie nature de cette agressivité : «... moi je venais de comprendre que je l'haïssais... avant j'osais même pas penser des affaires comme ça... hey !... haïr son père..." (p. 174). Par son exemple c'est encore Nathalie qui donne à Gaby le courage d'extérioriser son agressivité, “d'éliminer son père comme ça pffft!» (p. 176). "Parce que si on réalise rien, si on pose pas des actes importants ça vaut pas la peine de rêver... hein ?... ni même de vivre... " (p. 175). Alors le geste s'accomplit: Gaby frappe son père avec sa canne d'aveugle. Pourtant cette fois-ci la crise n'aboutit pas à un calme relatif.

L'acceptation du double comme une partie intégrale d'elle-même implique que Gaby est directement exposée à la mort et surtout à la folie. Dès le matin Gaby se rend compte de ce danger: «... je sens qu'il serait si facile de me laisser aller à ça non je ne suis pas folle non je ne vais pas devenir folle non non!» (p. 156). Plusieurs fois pendant la journée elle eut l'impression que ses pensées “se dérapaient" (p. 171, 193). Mais c'est surtout après la volée qu'elle donne à son père que Gaby doit confronter la mort et la folie. La chaleur symbolise l'angoisse existentielle que Gaby ressent: “... elle a excessivement chaud... comprimés calmants, oui tout prendre d'un seul coup avec un verre d'eau, hop! sauter la barrière... » (p. 191). La fin est tout près. Dans un dernier effort pour se sauver Gaby essaie de fuir mais elle se rend compte qu'il est trop tard. L'angoisse est devenue étouffante: "mon Dieu qu'il fait chaud! pas de bon sens, faire chaud comme ça non non, pas peur, Fœtus, ça ne peut pas te faire mal, un peu de chaleur, ce n'est pas grave, mais c'est bien trop chaud pour aller, travailler, pfiou !...” (p. 198). La chaleur en rappelant à Gaby fœtus et le 28 août semble fermer autour d'elle un cercle d'angoisse. Confrontée à l'image totale de son angoisse elle succombe et "ce fut tout noir, il n'y avait plus rien $»$ (p. 200).

Quand Gabrielle "raconte I'histoire de Gabrielle» (p. 205) elle prend plusieurs perspectives. Ces différentes perspectives s'expriment par un emploi très varié des pronoms personnels. Pour indiquer l'héroïne seule ou avec d'autres personnes, elle emploie les pronoms personnels suivants: je, tu, elle, on, nous, vous, elles, ils. Les pronoms je et elle sont employés le plus souvent. II y a une alternance régulière de ces deux pronoms.

Quand Gaby parle d'elle-même en compagnie d'autres personnes elle emploie on, nous, elles ou ils. Remarquons que l'emploi de nous ainsi que des équivalents de nous à la troisième personne elles et ils est rare. En parlant d'interaction entre elle-même et un(e) ou plusieurs autres person- 
nes, Gaby emploie on mais encore plus souvent elle évite l'emploi d'un pronom personnel. À cet effet elle sert de deux méthodes. Suivant la première elle sépare les actions de l'autre de ses actions à elle. Un exemple de ce procédé se trouve aux pages 106-112 où Gaby et Gloria vont au centre médical. Suivant l'autre méthode Gaby chosifie les personnes, y compris elle-même. Par exemple Gaby et sa famille se mettent à table: "Puis il y a le grand charivari qui précède le dîner, tout le monde se trouve en même temps dans l'entrée de la cuisine, ça s'agglomère compact on forme bouchon dans la porte..." (p. 133). Cette façon d'exprimer les contacts de Gaby avec les autres est à mon avis très significative. Elle indique clairement que Gaby ne connaît presque pas ce qu'on appelle en anglais un "we feeling ". Gaby se sent toujours isolée des autres, les relations interpersonnelles ne l'affectent pas vraiment. Dans cette perspective il est intéressant d'examiner de plus près les quelques exemples où l'interaction semble justifier l'emploi de nous, ils ou elles.

Nous ne s'emploie que quatre fois. Dans deux cas (p. 194, 199) il s'agit de Gabrielle et de Fœtus, Fœtus qui à ce moment-là est déjà mort depuis sept mois. Gabrielle vient de battre son père et est entré dans la lutte finale contre la folie, l'ultime conflit entre son double et son surmoi: «... non je ne veux pas, non je ne perds plus la boule... mais non t'es pas folle... oh non ne plus sortir de moi! non!..." (p. 195). Une partie de Gaby échappe à l'autre et elle se rend vaguement compte que c'est justement ce clivage du moi qui la mène à la folie. Elle sait par "instinct de conservation" (p. 197) qu'il ne faut "plus sortir du moi», qu'il faut unifier son moi. Je crois que c'est dans cette perspective qu'il faut voir le sentiment d'unité que Gaby a tout à coup et beaucoup trop tard avec fotus. Fœtus symbolise le double de Gaby et afin d'unifier son moi elle doit accepter ce double comme une partie d'elle-même. Désespérément elle essaie de maîtriser ce double qui, elle le sait, l'entraîne vers la folie.

Les deux autres fois que Gaby emploie nous, elle parle d'elle-même et de sa mère: a... nous sommes sorties dans le corridor... puis nous étions dans un grand vestibule..." (p. 208). La mère est à l'origine du surmoi de Gaby. En outre la mère est étroitement liée au «beau moi-même »: Nathalie. La mère est une "femme forte, oui faite pour encaisser les responsabilités... " (p. 124). Ceci m'incline à penser qu'on peut interpréter l'emploi de nous à cet endroit comme une note optimiste. Gabrielle se sent une avec sa mère, il y a un véritable contact entre elle et cette mère forte, ou en d'autres mots il y a prédominance du surmoi et identification entre Gabrielle et son «beau moi-mêmen.

I/s ne se trouve que cinq fois dans Apres la boue. I/s sont Gabrielle et son mari Roch. Le 28 août il y a beaucoup d'interaction entre Roch et Gaby. Pourtant ces cinq exemples (p. 41, 45, 74) sont les seules fois où Gaby unifie les actions de Roch et les siennes dans un ils. Dans tous les autres cas Gaby sépare ses actions de celles de Roch. Rien d'étonnant parce que Roch est beaucoup plus son ennemi que son mari. Gaby emploie 
ils quand sa relation avec Roch est protégée par la sauvegarde de la routine: le matin dans l'auto, et le soir dans la chambre à coucher. Je crois que la force de leur relation se trouve justement dans cette routine quotidienne, ces rites qui ne signifiaient rien ou qui signifiaient terriblement trop. 'C'est la routine qui a donné à Gaby le sentiment de sécurité, qui lui a fait supporter Roch, cette brute, pour cinq ans.

J'ai trouvé douze fois elles. Huit fois ce pronom désigne Gaby et Gloria Patry: «...elles avaient quatorze ou quinze ans... et elles marchaient à présent dans la ruelle, elles avançaient doucement dans l'ombre tiède de la ruelle... elles enjambèrent l'urine en retenant leur respiration et elles se retrouvèrent dans la cour de $M^{\text {me }}$ Punique... puis elles se faufilërent... et elles relevèrent leurs jupes... puis elles comparèrent leurs enfourchures... " (p. 29). Cet incident a été très important pour Gabrielle. Après les horreurs de l'oncle Émilien et du guenillou, Gloria Patry lui offre une expérience sexuelle positive. Gaby et Gloria sont deux jeunes filles qui vont essayer le fruit défendu: elles sont comme deux conspiratrices a... hypocrites se glissant comme des .spectres de fleurs dans la tourelle..." (p. 29). II y a unité d'action entre les deux filles. Dès qu'il s'agit de différence le mot elles disparaît: “... et c'était Gloria qui avait l'organe le mieux développé pour son âge... » (p. 29). Cela donne à Gloria une supériorité qui séparera les deux jeunes femmes pour toujours. (Cf. surtout p. 88-91 et p. 106-112.)

II n'y a qu'une autre fois où Gabrielle se sent si près d'une femme. Cette femme est sa cousine Nathalie qui lui ressemble. Cette ressemblance combinée avec les actes exemplaires fait de Nathalie un idéal aux yeux de Gabrielle. Elle l'appelle «ma sœur, le beau moi-même" (p. 176). La découverte de cet idéal du moi est très importante parce qu'elle va donner à Gaby la force de battre son père. C'est une découverte assez pénible qui ne se fait que graduellement. D'abord Gaby et Nathalie ne sont ensemble que par le fait qu'elles sont seules dans la même chambre: «...elles étaient à présent dans la chambre de Nathalie» (p. 170), puis elles se rencontrent dans la gêne: «elles se mirent à rire, pour rien au fond, peut-être parce qu'elles n'avaient rien à se dire..." (p. 171). Sauf par ces rires nerveux chacune continue à vivre dans son monde à elle. "Maintenant elle [Nathalie] est assise, elle aussi, au bord du lit, tout près de Gaby. - Sais-tu, Nathalie, je te regarde, puis je commence à trouver qu'on se ressemble... - Oui... oui, c'est vrai... on dirait deux sceurs... Elles se lèvent, puis se postent toutes les deux devant le miroir de la commode, se regardent... » (p. 172).

Une fois la découverte de la ressemblance faite un véritable contact semble possible. Gaby se rend compte que Nathalie a résolu des problèmes qui sont aussi les siens. Gaby semble s'identifier à Nathalie au point d'oublier pour quelques minutes ses pensées et son corps à elle. Malheureusement la "contagion de la santé morale " vient "trop tard"; Gaby est déjà une “emmurée vivante» (p. 176). Comme je viens de le démontrer Gaby n'a que très peu de contacts réels avec d'autres personnes. II s'ensuit que toute 
son attention se porte sur elle-même. Pour parler de la personnalité complexe de Gabrielle la narratrice emploie les pronoms je et elle. En général tous les aspects du moi sont indiqués par je. Le plus souvent je représente «le moi ordinaire et pour ainsi dire central» (B. p. 77) dans lequel les différentes facettes du moi collaborent plus ou moins paisiblement. Ce n'est qu'au moment d'une crise que cette unité se brise, que la collaboration s'arrête. II y a une scission à l'intérieur du moi et les deux parties qui se forment, le double et le surmoi, commencent un dialogue intérieur. Dans ce cas il arrive que le surmoi parle au double à la deuxième personne employant les pronoms tu et vous. Une autre façon de montrer la dualité du moi dans la langue écrite réside dans l'emploi du verbe pronominal. Dans le passage suivant je donne un exemple de l'emploi varié du pronom $j e^{8}$. Dans la "pénombre tiède» du Tréponème (p. 60) le je (o) est écarté et perd contrôle: a... je (o) ne suis pas capable de $m^{\prime}(0)$ arrêter, impossible, je (d) ris, je (d) ris, je (d) ris, je (o) voudrais bien cesser il n'y a plus de raison de rire comme ça, arrête(s)! arrête Gaby mais arrête-toi (d) donc!» (p. 61) Le double est dominant, même le surmoi n'y peut rien, "je (d) continue, je (d) suis lancée... et j' (s) ai honte mon Dieu que i' (s) ai honte!... à présent un waiter se penche sur moi...» (p. 61). Le waiter fonctionne comme un surmoi extérieur (NB. l'emploi des mots "sur moi»). Gaby s'évanouit: “je (o) flotte, je (o) me (d) sens toute légère, je (o) ne suis plus rien... salut je (o) pars» (p. 61). Le je ordinaire et central qui unit toutes les facettes de la psyché est “annulé en douceur» ( $p$. 61), la douceur déceptive du je (d): “le temps $m^{\prime}$ (d) effleure et passe sur moi sans $m^{\prime}$ (d) entamer" (p. 62). Les mots "sur moi introduisent l'éveil du je surmoi. A partir d'ici Gaby commence à redevenir «vaguement lucide... faces fouineuses penchées sur moi, ça (d) me (o) cerne tout autour je (o) vais étouffer si ça (d) continue, puis je (o) me (d) sens redevenir pesante, je (d) rentre en moi (o)...” (p. 62).

Une domination prolongée du double sera insupportable: «je vais étouffer». A l'aide du surmoi le double "rentre en moi", le double redevient un aspect du moi ordinaire qui a repris contrôle: «je (o) sens que je (o) vais de nouveau être bien, oh très bien je (s) veux (p. 62) ». Le retour du moi (o) implique le retour des questions auxquelles Gaby préfère ne pas répondre. Pourquoi a-t-elle vomi? «... au fait c'est fou de vomir comme ça... les nerfs encore?... ou enceinte pour vrai ? (p. 63). Gaby craint d'être enceinte, voilà la surface d'une angoisse qui comprend l'angoisse du double que sera cet enfant et le dégoût qu'elle ressent pour son mari: “...ça (d) y est je (o) suis mère, non!... VOUS (o) VOUS (o) PERPÉTUEREZ À TRAVERS VOS ENFANTS VOUS (0) VIVREZ EN EUX MÊME APRÈS VOTRE MORT ET N'OUBLIEZ PAS QUE LES ENFANTS CONSOLIDENT L'AMOUR DU COUPLE... mais non je (d) ne veux pas... non je (d) ne veux plus mentir c'est assez! MAIS VOUS (o) NE COMPRENEZ DONC PAS QUE ROCH JE (d) LE DÉTESTE!... voyons! mais qu'est-ce que je (d) dis là? non je (s) ne veux pas $j$ ' (s) aime mon mari $j$ ' (s) aime mon mari $j^{\prime}(s)$ aime mon mari!..." (p. 63) Le double et le surmoi s'adressent au je ordinaire: vous. C'est comme un plaidoyer entre deux parties: le surmoi 
et le double. Le je (o) fonctionne comme juge. Cette impression est renforcée par le ton officiel, l'emploi de vous (d'habitude on se tutoie) et l'expression "je le jure" (p. 63). Après avoir juré qu'elle aime son mari, la réalité qui l'entoure s'impose et Gabrielle redevient elle.

Reste à répondre à la question: «Pourquoi la narratrice passe-t-elle de je à elle ? Raconter son histoire aux autres malades forme pour Gabrielle la dernière phase dans la recherche du moi. Après avoir éliminé ses complexes elle s'était totalement perdue dans le puits de la folie. Sortir de ce puits est comme une renaissance: Gabrielle doit redécouvrir qui elle est. En faisant continuellement revivre son passé, elle espère découvrir peu à peu son moi authentique. L'héroïne est la Gabrielle du passé et la narratrice la Gabrielle actuelle. Dans cette perspective l'emploi du pronom pourrait être très révélateur. Quand la narratrice emploie je elle reconnaît dans l'héroïne un aspect authentique d'elle-même. L'emploi d'elle est un signe d'aliénation entre la narratrice et l'héroïne. La narratrice ne se reconnaît plus dans l'héroïne. En considérant qu'elle s'emploie surtout pour rappeler les actions de Gabrielle et je pour faire revivre ses pensées et ses émotions, il est compréhensible que je soịt plus près de la narratrice qu'elle. Beaucoup plus que les pensées et surtout que les émotions, les actions sont souvent motivées par l'habitude et l'opinion des autres. Aussi les actions ne correspondent pas toujours aux désirs du moi profond et authentique. Ceci explique que Gabrielle la narratrice ne peut plus s'identifier à celle qui a réalisé ces actions dans le passé.

Bien que dans la plus grande partie du livre, il y ait une alternance régulière entre je et elle, il existe quelques passages qui sont exclusivement en je ou en elle. 1. Les passages au temps de l'écriture sont en je. La narratrice parle d'elle-même au présent. (p. $14,15,18,22,23$ ) 2 . Le résumé (p. 18-22) où la narratrice veut se distancier de l'héroïne, peindre I'histoire d'une autre est presque exclusivement en elle. Cette manière de raconter sans identification émotionnelle semble mal réussie, il n'y a pas beaucoup à dire: «Non... je vais trop vite» (p. 22) 3. Les passages les plus significatifs pour l'emploi du pronom se trouvent dans la postface (p. 200-208). Dans la première partie de la postface (p. 200-204) elle domine tandis que la deuxième partie est complètement en je. Je crois que dans ce passage l'emploi du pronom reflète la santé mentale de Gabrielle. Au début tout "fut noir» ( $p .200$ ) et la narratrice ne s'en souvient pas très bien: «je me souviens mal» (p. 201). Gaby est perdue dans la folie. II est compréhensible que la narratrice ne s'identifie pas à cette femme folle. Le passage de elle à je correspond au moment où Gabrielle se sent guérie: «... elle marchait dans... une cour,... et brusquement je me sentis prisonnière et je crus comprendre ce qui $m$ 'arrivait et je me sentis tout à fait revenue à moi et guérie..." (p. 204). Le fait que les dernières pages soient totalement en je me laisse avec un sentiment optimiste sur le sort de Gabrielle. La narratrice semble avoir réussi à se réconcilier avec l'héroïne et à trouver son moi authentique. 


\section{Les trois premiers romans}

Il existe un lien étroit entre le Nombril, Corridors et Après la boue ${ }^{9}$. Bien que l'auteur présente trois personnes différentes (deux hommes et une femme) dans trois aventures différentes, les problèmes qui hantent ces trois héros se ressemblent tant qu'on se demande s'il ne s'agit pas des mêmes problèmes que chacun essaie de résoudre à sa manière. Dans ce cas une autre question s'impose: $y$ a-t-il du progrès ? est-ce que l'héroïne d'Après la boue s'approche plus de "la solution" que les héros des deux premiers romans?

Avant de chercher une réponse à ces questions je veux brièvement présenter ces deux héros. Jérôme, le héros du Nombril, a vingt-deux ans $(N, p .24)$ et vit avec sa mère et son frère Marcel dans un appartement à Rosemont. II a une petite amie, Nathalie, qui réapparaîtra dans Après la boue comme la cousine de Gabrielle. On suit Jérôme pour une période d'un peu plus de vingt-quatre heures. Au début de cette période il se fait congédier pour acquérir une liberté qu'il qualifie presque immédiatement après d'“accidentelle et provisoire, sans plus, fausse liberté d'ailleurs..." $(N$, p. 49). Pendant les heures qui suivent il essaie d'assumer cette libération "si exigeante, si vaste et si déserte..." ( $N$, p. 59). Dans Corridors le protagoniste s'appelle Clément. L'histoire de Clément, qui dure un peu plus d'un an, commence quand celui-ci a vingt-trois ans. $\dot{A}$ cet âge il quitte la maison paternelle, qui se trouve aussi à Rosemont, pour «vivre au grand soleil» ( $C$, p. 103). Clément, lui aussi, «sent... le vide de cette illusoire liberté" ( $C$, p. 13). Contrairement à Jérôme il se dirige vers les autres pour combler son vide. Il se marie avec Céline qu'il quittera quelques mois après le mariage. Alors “pour ne pas sombrer... Clément s'engage[ra]... dans [un] sous-groupe lié aux activités du F.L.Q." (C, p. 13). Dans le Nombril et Corridors, comme dans Après la boue, le héros commence par poser un acte de rupture pour obtenir la liberté, " pour devenir ce qu'[il] doit être" (C, p. 180). De quoi les trois héros veulent-ils se libérer par cette ru-ture? De "ce passé... de plus en plus lourd... [qui] colle au dos comme une boue glacée... " (AB, p. 15). Ce passé c'est l'enfance, évoquée dans les trois livres sous forme de souvenirs. Dans les trois cas le dernier souvenir d'enfance remonte à la quinzième année. De quinze ans jusqu'au début de l'histoire proprement dite il ne se passe quasiment rien. Quinze ans marque le début de l'adolescence dans l'œuvre de Larocque: “... et je finis, moi aussi, par avoir quinze ans... j'avais glissé insensiblement dans l'adolescence... " ( $C$, p. 101). A quinze ans se produit aussi une aliénation du moi ${ }^{10}$, on commence "à ne plus vivre qu'autour du moi..." (C, p. 101). Afin de retrouver le moi perdu, le héros doit sortir de la routine quotidienne, il doit rompre «avec soi pour mieux se retrouver" $(C, p .143)$. II me semble que les trois romans racontent trois manières différentes d'accomplir ce processus d'auto-recherche:

1. "Ou bien on comprend tout ça, on se sent tout obligé, mais on n'a pas la force de réagir, c'est trop difficile, on reste comme on est, et on 
continue de vivre seulement pour soi, la face collée sur son petit nombril, on continue de croire que la liberté c'est de faire tout ce qu'on a envie de faire et rien que ça, c'est-à-dire qu'on ne peut plus le croire comme avant, mais on fait semblant, toujours la bonne conscience !... » (C, p. 181)

2. "Ou d'être tout simplement soi-même avec tout ce que ça comporte, de s'intégrer dans un bloc social qui nous forcerait à sortir de soi et à vivre pour et avec les autres...» $(C$, p. 181)

3. Ou «on vieillit dans son mensonge comme une mouche dans les ordures... et vient un temps où on avait envie de cracher à la face, de s'autovomir... on aperçoit qu'on est resté au pied de l'escalier, qu'en s'épuisant sur la première marche sans se donner la pleine de monter..." (C. p. 181).

Le premier extrait résume l'aventure de Jérôme. Jérôme finit par faire semblant d'être maître de son sort dans “un jeu» (N, p. 209), mais il se rend en même temps compte des limites de son jeu car il ajoute "comme si c'était toujours à lui seul de décider...» (N, p. 209); «... il sait bien que son mal est en lui, quelque part au fond de lui, si profondément enraciné qu'il est devenu un peu lui-même, indiscernable d'avec lui-même... oui le salut c'est les autres, héhé! l'amour, sortir de soi pour prendre possession de soi, oui... (N, p. 191).

Ainsi, à la fin de son aventure, Jérôme annonce l'aventure de Clément qui se tournera vers les autres (cf. ci-dessus 2.). Clément décidera à la fin de son expérience de retourner vers sa femme Céline parce que malgré tout il croit toujours que l'être humain ne peut se trouver que dans l'amour. D'où va venir l'amour dans ce mariage mal réussi? «L'amour viendra bien tout seul puisqu'il sera déjà contenu dans ses actes. Le combat est fini... mais il n'y a pas eu de combat, il avait toujours été gagnant. II frissonne un peu..." (C, p. 148). Clément semble penser qu'il a réussi à se libérer mais en même temps il se rend compte que rien n'a vraiment changé: "il avait toujours été gagnant". Est-ce que son espoir, sa confiance que l'amour "viendra tout seul " sont faux? S'il faut en croire Gabrielle la réponse est oui. Pendant sa nuit de noces Gabrielle aussi est encore confiante "puis je l'aime après tout, non? puisque je l'ai épousé [acte] ça prouve tout..." ( $A B$, p. 36). Mais le 28 août elle découvre que "ce fameux amour! [est] un absurde mensonge " $(A B, p .63)$ ce qui rappelle le troisième passage cité plus haut: "on vieillit dans son mensonge comme une mouche dans les ordures...". Le 28 août Gaby comprend "qu'[elle] est resté[e] au pied de l'escalier" (C, p. 181). Chacun des trois romans commence par une libération. Une fois cette liberté acquise elle paraît limitée. La limite de la liberté forme le point de départ du roman suivant. Jérôme se libère de la pression extérieure pour faire ce qu'il a envie de faire, mais finit par se demander s'il peut vraiment se passer des autres. Clément quitte la maison paternelle pour se trouver dans une vie "pour et avec les autres" (C, p. 181). II finit par espérer que l'amour, la base d'une telle vie, viendra tout seul. Gaby a découvert que l'amour ne vient pas tout seul et c'est elle qui subit "ce tourment bien plus terrible de se pardonner - et non de 
s'excuser - et de se réconcilier avec soi-même...» $(C, p .147)$ que Clément prévoit en cas d'échec. Les trois efforts pour trouver le moi authentique peuvent donc être considérés comme une suite. On pourrait même parler d'évolution s'il était possible de démontrer que c'est le passé qui empêche chacun des héros "de se réconcilier avec soi-même" ( $C, p .147)$. Les trois héros commencent par couper les liens avec leur passé mais chacun d'entre eux se rendra compte que "tout cela c'était lui » $(N, p .207)$ et qu'il faut «vivre avec ce passé et malgré lui » (AB, p. 205 et aussi $C$, p. 147). J'ai démontré que pour Gaby le passé, l'enfance, forme la base de ses complexes et de ses problèmes. II me semble donc que pour savoir s'il y a des points communs dans les problèmes des héros il faut se tourner vers les souvenirs d'enfance qui forment d'ailleurs une partie importante des trois romans.

Si l'on examine le rôle des parents on se rend compte que l'influence du père est réduite à zéro ou presque lorsque l'enfant a quinze ans. Le père de Jérôme meurt quand celui-ci a quinze ans $(N, p$. 9). Clément se rappelle qu'à quinze ans son père «apparaissait... après des années et des années de comédie, comme un être faible et démuni et solitaire et vide" (C, p. 102). Le père de Gaby devient aveugle quand celle-ci a quinze ans ( $A B$, p. 102). En conséquence de la quasi-disparition du père, le rôle de la mère acquiert de l'importance. Les mères de Jérôme et de Gaby vont gagner la vie pour la famille $(N$, p. 144, $A B$, p. 98$)$, tandis que le père de Clément "laiss[e] peu à peu la boussole familiale se figer vers maman" $(C$, p. 100). Avant le quinzième anniversaire des héros, le père a joué un rôle essentiel dans leur vie. J'ai déjà montré l'importance du père de Gabrielle dans la formation de ses complexes. Jérôme est obsédé par la mort $(N$, p. 105) et surtout par la mort de son père dont le souvenir lui revient très souvent. Cette obsession pourrait indiquer qu'il avait des sentiments ambivalents à l'égard de son père. Cette ambivalence se révèle dans la frustration sexuelle de Jérôme qui aboutit à l'impuissance quand il fait l'amour avec Nathalie ( $N$, p. 177). Nathalie par contre éprouve un grand plaisir (cf. Gabrielle et Roch).

Par nature le père de Jérôme est son prototype, son exemple de virilité, mais en même temps il a peur d'être castré par lui. Cette angoisse apparaît surtout dans deux scènes qui ont leur parallèle dans Après la boue. "ll y a des hommes noirs qui enlèvent les enfants, gare aux vieux fifis! pédérastes aux ongles sanglants, vampires souriants, entants ramassés par de sadiques vivisecteurs qui vous dépècent en un rien de temps le petit corps violé... garçons au sexe coupé net comme une fleur molle sa tige sectionnée au niveau du bas-ventre avec un rasoir comme celui de papa, la castration rôde autour de ton lit, nuits poisseuses cauchemars rouges de l'enfance maman! maman !... ( $N$, p. 74,75 ; cf. $A B$, p. 8,9 et 97 ) II faut noter surtout l'association entre le rasoir menaçant et le père. Comme Gaby ( $A B$, p. 97), Jérôme demande du secours à sa mère. Dans la deuxième scène du Nombril Jérôme demande le soutien de son père: “on s'en retourne chez nous, hein papa? tous les deux, tu t'en viens chez nous ?... - Non... 
retourne jouer avec les fleurs. Merci, merci... mais je ne peux pas, l'arbre va tomber d'une minute à l'autre et quand l'arbre sera tombé ce ne sera plus le temps de jouer avec les fleurs, tu le sais bien... et c'est presque la nuit, papa! la nuit, et j'ai peur de la nuit... je vais avec toi... - Non, j'ai dit d'aller jouer... tiens, amuse-toi avec cet œuf... Un œuf noir dans ma main... non! non! non! pas l'œưf noir... où es-tu papa! j'ai peur des bûcherons l'arbre n'en peut plus... l'arbre frémit à chaque coup de hache ça lui fait mal oh que ça fait mal attention! on penche! attention! tombe du côté où il penche, arrêtez je tombe, je tombe, j'arbre-tombe. » (N, p. 127) Comme le père de Gaby, le père de Jérôme refuse d'accorder du soutien à son enfant. Il laisse Jérôme avec un œuf noir qui semble symboliser le double de Jérôme et il ne fait rien contre la coupe de l'arbre (cf. $A B$, p. 85, 98).

Ces exemples démontrent clairement qu'il y a des points communs entre les complexes de Gaby et de Jérôme. Jérôme semble avoir des frustrations sexuelles comparables à celles de Gaby. L'histoire du Nombril semble correspondre à celle du 28 août dans Après la boue. Au cours du roman Jérôme essaie de quitter Nathalie, comme Gaby quitte Roch. Contrairement à Gaby il n'y réussit pas. Finalement c'est Nathalie qui s'en va. L'explication de cet échec, semble-t-il, c'est que le double de Jérôme ne s'est pas encore développé, ce n'est qu'un «œuf noir». Gaby commence à agir quand son double est devenu une entité palpable sous la forme de fœtus. Cette forme du double rend un rejet possible. Jérôme n'en est pas encore là, il s'identifie au fœetus, ce n'est pas un autre c'est lui-même $(N, p .123$, 133). Dans Corridors le double est symbolisé par Rosaire, le frère de Clément. Rosaire ressemble à fœetus, au père de Gabrielle et aussi à la tante Éva, donc à toutes les formes du double de Gabrielle.

Comme la tante Éva se balance dans sa chaise $(A B, p$. 9) Rosaire «Se balance toujours" $(C$, p. 73$)$. La tante Éva «buvait à même le goulot» $(A B$, p. 11,12$)$ et Rosaire "fumait... comme un bébé qui tête" $(C, p .70)$. $D$ 'ailleurs tous les deux sont fous bien que de façon différente. L'action de boire se retrouve aussi chez le père de Gaby qui boit sa bière "une bouteille après l'autre» $(A B, p .131)$. Rosaire a encore plus en commun avec "popa». Ni l'un ni l'autre ne parlent proprement. Rosaire mâche "des mots de fou que personne comprend", ou pleurniche $(C, p$. 78). Le père de Gaby n'est «pas capable de parler comme un homme: grognements confus... II secoue sa tête, son air d'enfant qui va pleurer... " (AB, p. 154). Rosaire et le père de Gaby sont tous les deux comparés à un chien. Rosaire est «inoffensif comme un grand chien jaune" $(C, p .73)$ et "popa" a un air de "chien battu" $(A B$, p. 154). Quand Gaby craint que son père soit mort, elle fantasme: «il a toujours dans la face son petit sourire de rien du tout... et il meurt tout seul. comme un chien..." ( $A B$, p. 100). Clément décrit son frère tué par une voiture en ces termes: «... il a encore son sourire qui veut rien dire... c'est vrai qu'il a l'air d'un grand chien jaune..." (C, p. 93)

La ressemblance entre Rosaire et Fœtus saute aux yeux. Du point de - vue physique Clément note qu' «on dirait qu'il [Rosaire] a la tête trop pe- 
sante” ( $C$, p. 78), une des caractéristiques d'un fœtus. Mais c'est surtout par sa mort précoce que Rosaire ressemble à fœtus. Rosaire meurt a avant d'avoir vécu, mort inutilement comme il avait inutilement vécu - la mort des idiots" $(C, \mathrm{p}$. 121). C'est "comme si ce cadavre de quinze ans ou à vrai dire sans âge, n'avait jamais cessé d'être cadavre» $(C, p .93)$. Lors de l'avortement, fcetus meurt aussi "avant d'avoir vécu». Quand Gaby va tuer fœtus elle dit tout bas: «ll le faut» $(A B$, p. 140). Clément se souvient d'avoir pensé quelque chose comme: "Rosaire s'est fait tuer, fallait que ça arrive" $(C$, p. 92), lorsqu'il a vu son frère mort. Il semble donc que ces deux morts étaient inéluctables et nécessaires. La relation entre Clément et Rosaire ressemble à celle entre Gabrielle et fœtus sous plusieurs aspects. Comme Fotus Rosaire est incapable de se défendre. Clément se sent responsable pour "mon Rosaire" (C, p. 74). Mais si Gabrielle éprouve Fœtus comme un fardeau qui est source d'agressivité et de haine, Clément voit dans ses activités protectrices une source d'amour: “... et moi, je l'avais gagné, le droit d'aimer Rosaire avec mon cœur, parce que je savais déjà l'aimer avec mon corps et avec mes souffrances et avec mes petites persécutions et avec tout ce que j'étais, le meilleur comme le pire..." (C, p. 182).

Pourtant Rosaire continue à hanter Clément. Même après sa mort il pense souvent à lui, surtout à «ce Rosaire du dernier jour»; il essaie "de comprendre, et [il] en arriv[e] invariablement à [se] dire qu'il n'y a rien à comprendre " $(C$, p. 101). C'est au fond le souvenir de Rosaire qui donne à Clément la force d'échapper à sa mort à lui. Clément est sur le point de glisser dans la mort quand " tout à coup il sursaute... ne pas mourir comme un chien (en une seconde il a revu, perdu en un repli douloureux de son enfance, le pauvre cadavre de Rosaire...) Non, pas moi! " (C, p. 121).

Par sa mort Rosaire sauve la vie de Clément et dans un sens Fœtus assure aussi le salut de Gaby. L'après-midi de l'avortement Gaby a une forte tendance suicidaire $(A B$, p. 136), mais au lieu de se suicider elle tue Fœtus. C'est encore grâce à Rosaire que s'expliquent les relations entre Clément et son père. Toutes les interactions entre Clément et son père ont un lien avec Rosaire. Le père punit toujours Rosaire même quand c'est Clément qui est coupable $(C, p$. 74). Quand le père punit Clément c'est parce qu'il a défendu Rosaire $(C$, p. 80 ). II y a deux moments où Clément semble se sentir près de son père, une fois après l'accident fatal de Rosaire et la deuxième fois après qu'il a failli mourir lui-même. On pourrait dire que le père de Clément a produit deux doubles: un positif, Clément, et un négatif, Rosaire. II a honte du double négatif et il ne veut rien en savoir, tout comme le père de Gaby ne veut rien savoir de Gaby quand elle fait des actions inspirées par son double. Quand Gaby a quitté Roch son père refuse d'écouter son histoire $(A B, p .87)$ et quand elle s'est fait avorter son père feint de l'ignorer: «tabou, ça» $(A B$, p. 152). Pourtant Gaby dit: “... je suis sûre qu'il souffre de moi, ou souffre de lui à travers moi...» $(A B$, p. 147). La mort de Rosaire est gardée sous un même silence, « maman s'arrangeait pour parler d'autre chose...» (C, p. 98). On pourrait pousser beaucoup plus loin l'analyse des relations entre Clément, Rosaire et leur 
père, mais je crois avoir suffisamment démontré les analogies qui existent entre Rosaire et Fœtus. Rosaire est l'obsession principale de Clément, obsession qui est étroitement liée a ses sentiments à l'égard du père. Gaby, fœetus et le père forment un triangle affectif analogue. Aussi me semblet-il juste d'affirmer que Corridors correspond à la deuxième partie d'Après la boue comme le Nombril correspond à la première partie.

Maintenant il est clair que dans les trois romans il s'agit non seulement de trois personnages qui essaient de trouver leur identité, mais qu'il y a aussi une relation étroite entre les complexes qui poussent à cette recherche du moi authentique. La constellation des complexes de Gabrielle semble valable pour expliquer les trois livres. L'angoisse et la haine découlent d'une expérience "frustrante " avec le père. L'angoisse se fixe dans une obsession sexuelle. La haine est refoulée dans un double. Le Nombril raconte un effort pour résoudre l'obsession sexuelle. Corridors raconte un effort de réconciliation avec le double. Dans Après la boue l'auteur pénètre jusqu'au fond du problème en refaisant les deux premiers pas pour aboutir à la source des complexes: le père.

II y a donc évolution. Dans chaque roman le héros découvre un peu plus sa personnalité authentique. Cette évolution se reflète aussi dans les titres et dans l'emploi des pronoms personnels. La suite des titres représente les stades symboliques d'une naissance. La phase de la naissance symbolisée par le titre du livre correspond au progrès que son héros réalise. Le Nombril représente la matrice. Jérôme n'en sort pas, il reste «solitaire replié sur [son] nombril» ( $N$, p. 105). Il ne se sent pas la force "de couper le cordon ombilical » $(N, p .73)$, de quitter la maison «maternelle». Clément dans Corridors (le passage du stade intra-utérin au monde extérieur) «s'est amputé... de son cordon ombilical» $(C, p$. 143). II a quitté la maison paternelle et il essaie de se frayer un passage vers l'extérieur de différentes manières (Corridors). Gaby fait encore plus de progrès, elle passe par la boue (le cloaque). Gaby semble être (re)née mais il n'est pas encore clair que le nouveau-né soit viable.

Je crois après avoir étudié l'emploi des pronoms dans ces trois romans qu'il faut se tourner vers leur thème central: la recherche du moi authentique. La recherche du moi implique l'existence d'au moins deux moi : le moi cherchant et le moi cherché. L'existence de plusieurs personnalités dans une personne invite à une expression de cette pluralité au moyen des pronoms personnels. Si l'on admet que dans les trois romans le narrateur est en même temps le héros, le je représente une identification du narrateur au héros et le il une séparation des deux. Le narrateur est le moi cherchant qui reconnaît parfois dans le héros le moi cherché (je) et parfois considère le héros comme un étranger (ii). En acceptant ces idées de base, l'emploi du pronom personnel devient très significatif.

Si on projette l'emploi du pronom sur une ligne qui représente la vie des différents héros on peut en induire le progrès que le narrateur/ héros fait dans la recherche de son identité. Dans le Nombril il y a de longs passages exclusivement à la troisième personne, notamment les 


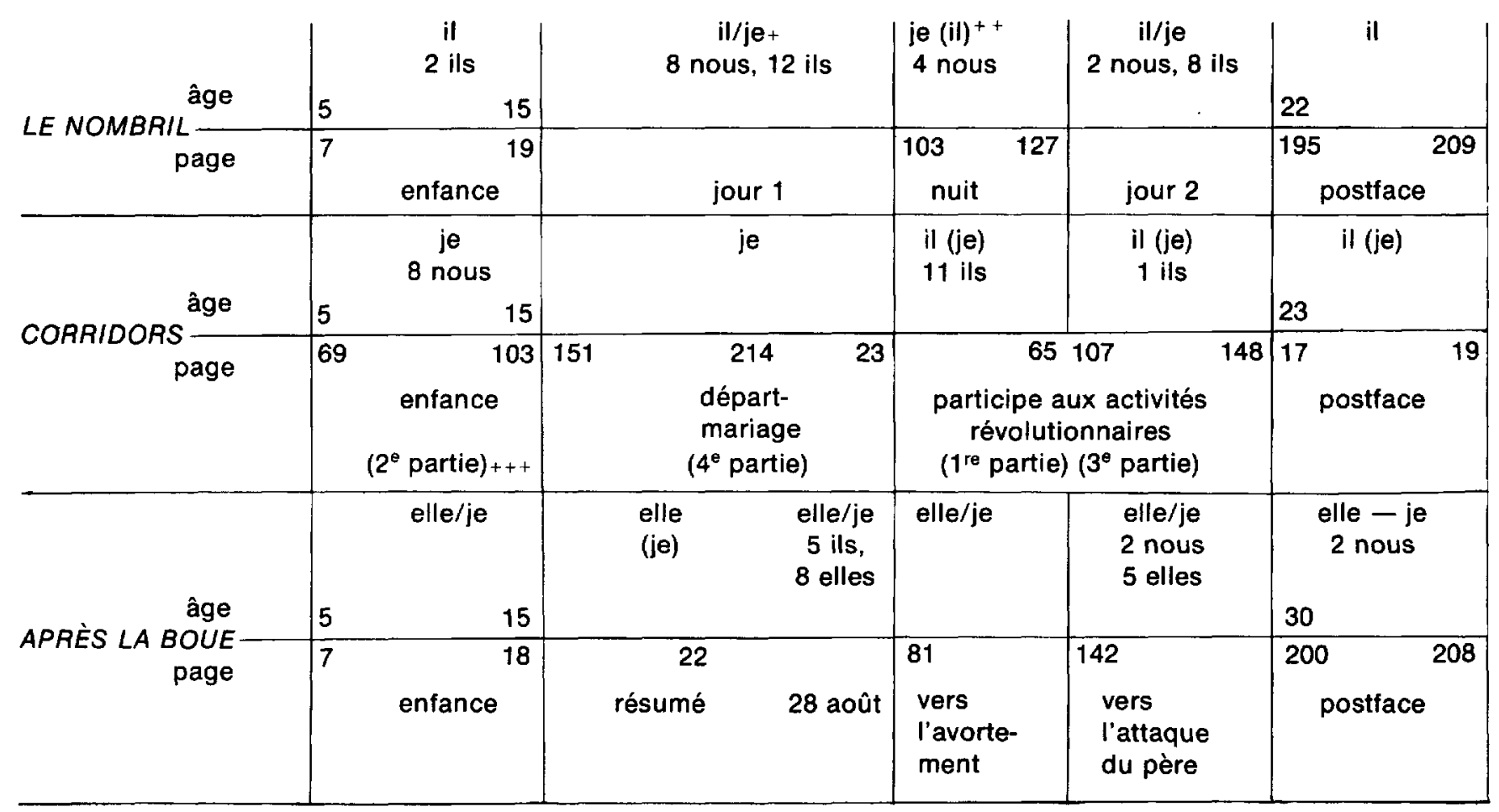

$+\quad i l / j e:$ une alternance régulière entre $i l$ et je

$+\quad$ il (je): il est dominant mais il y a quelques rares cas de je

+++ la suite des parties est réarrangée pour obtenir un ordre chronologique. 
souvenirs d'enfance. Jérôme ne réussit pas à assumer son passé comme faisant partie de son psychisme. Pendant les heures où il essaie de trouver son moi authentique il y a alternativement identification (je) et séparation (il) entre le narrateur et le héros. Le seul épisode prolongé où il y a identification entre les deux se situe dans la maison abandonnée où Jérôme et Nathalie passent la nuit. Le seul il dans cette partie se trouve tout au début du passage. Très vite Nathalie s'endort. Jérôme passe une nuit blanche remplie d'un mélange de fantasmes, de souvenirs et de cauchemars. Dans cet état demi-conscient, le narrateur et le héros se confondent (je). L'emploi des pronoms personnels pluriels est le plus fréquent dans le Nombril. II y a seize nous et vingt ils. Bien qu'ici comme dans les deux autres romans le narrateur semble éviter les pronoms personnels pluriels en séparant les actions du héros de celles des autres ou en employant on, Jérôme, faute de trouver son identité (je), tend à fuir dans le nous/ils. J'emploie le mot "fuir" parce que je crois que le sentiment d'unité avec les autres (notamment avec Nathalie) est faux. Jérôme n'a pas encore trouvé l'unité à l'intérieur de lui-même, comment peut-il donc se lier aux autres? II s'en rend compte lui aussi: «non, je ne connais pas Nathalie, c'est une étrangère pour moi, je vis avec des étrangers, ma mère et mon frère me sont inconnus... je ne connais que moi, et encore !..." $(N$, p. 100)

Dans la postface, où Jérôme le narrateur présente une évaluation de son expérience, il n'emploie ni le je ni des pronoms personnels pluriels. II continue à être $i l$, un autre. II n'y a pas d'identification finale entre le narrateur et le héros; Jérôme ne réussit pas à trouver son identité.

Clément dans Corridors a peut-être un peu plus de succès. II n'y a aucun passage exclusivement en il bien que dans deux parties la troisième personne soit nettement dominante. II faut d'ailleurs noter que la division formelle de ce livre a amené un emploi plus régulier des pronoms personnels. Grosso modo il y a deux parties à la première personne et deux parties à la troisième personne. La deuxième et la quatrième partie (chronologiquement la première et la deuxième partie) sont totalement en je. Clément le narrateur s'identifie à l'enfant Clément (je) et il a un sentiment d'unité avec sa famille ( 8 nous). Quitter la maison paternelle semble refléter un désir authentique du héros (je), mais il est seul maintenant: il n'y a aucun nous dans cette partie. Clément a «rompu avec soi pour mieux se retrouver (peut-être dans les autres)" $(C, p, 143)$. Le doute exprimé par le mot "peut-être " paraît justifié ; à partir du moment où Clément se cherche dans les autres tout tourne mal. $\grave{A}$ la fin de la quatrième partie il rencontre sa femme Céline, plus tard il se lie à une cellule révolutionnaire. Ces relations avec “les autres" l'entraîne à ne vivre qu' «autour de [soi]» (C, p. 101). L'aliénation du moi authentique est exprimée au niveau pronominal par il. Au début de cette période ( $C$, p. 23-65), Clément a encore des contacts qu'il croit valables, notamment avec son ami Jean-Louis (11 ils). Dans la troisième partie qui chronologiquement suit la première, Clément rend impossible son appartenance au groupe révolutionnaire. Ce groupe le rejette et essaie de le tuer. De nouveau Clément est seul. Dans la troisième 
partie ils n'apparaît qu'une seule fois. Ce pronom désigne ici Jean-Louis et Clément mais la phrase marque la fin de leur amitié: "De toute façon, ils s'étaient évidemment reniés l'un l'autre depuis cette fameuse nuit..." $(C, p .134)$ Dans la postface $(C, p .7-19)$ Clément n'a pas encore trouvé son identité mais il semble l'entrevoir. La postface est en il mais avec quelque hésitation, semble-t-il, le narrateur introduit quelques je: «il pense: je...»; “il se dit: je...» $(C$, p. 10).

Dans l'œuvre de Larocque, où l'on passe sans s'en rendre compte de la première à la troisième personne, ces petites phrases d'introduction (il pense, il se dit, etc.) semblent un miroir de l'incertitude du héros. Clément croit avoir trouvé son vrai moi mais en même temps il pressent vaguement que ce n'est qu'un mirage destiné à disparaître rapidement. Malgré ce pressentiment, il m'apparaît que Clément est plus près de son but que Jérôme. Dans le Nombril le narrateur ne peut s'identifier au héros que quand celui-ci est dans un état demi-conscient. Le narrateur de Corridors par contre semble avoir accepté le passé du héros, notamment son enfance, comme une partie authentique de sa personnalité. Dans Après la boue l'emploi des pronoms donne vraiment lieu à de l'espoir. À travers tout le livre, y compris les souvenirs d'enfance, il y a un mélange de je et de elle. Dans la postface la narratrice est d'abord complètement aliénée de l'héroïne (elle) qui a glissé dans un état de folie. Le début de la convalescence coïncide avec l'identification entre la narratrice et l'héroïne (je). Cette fusion entre le moi cherchant et le moi cherché promet d'être permanente. Elle est encore affirmée par le double emploi de nous. Maintenant que Gaby a retrouvé son identité, elle peut avoir des contacts réels et valables avec d'autres êtres humains.

La présente étude des trois premiers romans de Gilbert Larocque combine une analyse du caractère des héros avec une analyse pronominale. L'analyse des pronoms personnels est une forme d'analyse assez peu explorée. Je ne suis pas sûre que l'analyse pronominale puisse servir comme méthode autonome mais à la suite de cette étude je suis convaincue que l'emploi des pronoms personnels peut donner des indications très valables sur:

1. la relation entre le narrateur et le protagoniste du roman. Le choix du pronom qui désigne le personnage principal éclaire, surtout s'il y a, comme dans les romans de Larocque, une fluctuation dans l'emploi de ce pronom, l'attitude du narrateur vis-à-vis de ce personnage. Si l'on peut suivre cette attitude à travers plusieurs livres d'un même auteur, des renseignements intéressants sur la personnalité du narrateur peuvent en être tirés.

2. le caractère du protagoniste.

a) Dans un dialogue intérieur le héros peut employer la deuxième personne (tu ou vous) pour se parler à lui-même. Ceci peut révéler un clivage 
de sa psyché qui autrement passerait inaperçu. L'emploi de certains verbes pronominaux peut aussi témoigner d'une scission psychique.

b) Le héros peut se distancier de lui-même en employant la troisième personne (il ou elle). Ceci peut être un signe d'aliénation entre le héros et certaines de ses activités physiques ou affectives.

c) L'emploi des pronoms personnels pluriels ou leur absence nous renseigne sur la valeur que le héros attache à ses relations avec les autres.

Dans l'étude d'Après la boue l'analyse pronominale m'a aidée à mieux comprendre le fonctionnement de la psyché de Gabrielle, surtout pendant les crises. Dans l'analyse diachronique des trois premiers romans de Larocque, elle m'a fait mieux apprécier l'évolution qui existe dans son œuvre. Le thème central de cette ceuvre paraît être la recherche de l'identité. L'identité est masquée par des complexes qui proviennent d'expériences non assumées. Dans le Nombril, Corridors et Après la boue le héros essaie d'enlever ce masque. Contrairement aux héros du Nombril et de Corridors qui ne détruisent qu'une partie du masque, l'héroïne d'Après la boue semble avoir éliminé tous les obstacles qui l'empêchaient de voir son moi authentique. Elle semble prête à commencer une vie qui est en harmonie avec ce moi retrouvé. Est-ce que le protagoniste réussira vraiment à assumer son identité et à commencer une vie nouvelle? Après la boue nous laisse dans l'incertitude là-dessus. En lisant le dernier roman de Larocque, Serge d'entre les morts, j'ai essayé de trouver une réponse à cette question. Ma première impression est que Serge d'entre les morts nous procure en effet cette réponse. Le protagoniste semble avoir assumé son identité. Il a intériorisé les fantômes familiaux de son passé "que rien ne pourrait jamais exorciser " (Serge, p. 148). Ils sont devenus une partie intégrale de sa psyché: il y a une identification complète entre le narrateur et le héros (je).

La vie nouvelle, annoncée sous forme de voyage (Serge, p. 68-70 et 141-142) se situe toujours dans l'avenir et peut-être même ne commencerat-elle jamais: "car je savais... que je n'avais pas trop de toute une vie (comme si le jeu en valait la peine) de briser le cercle des perpétuels recommencements" (Serge, p. 148).

Els Post-Pieterse

1. Montréal, VLB éditeur, 1976.

2. Montréal, Jour, 1970.

3. Montréal, Jour, 1971.

4. Montréal, Jour, 1972.

5. Charles Baudouin, lÂme enfantine et la psychanalyse, Neuchâtel, Delachaux et Niestlé, 1964. Dorénavant je désignerai ce livre par le sigle $B$.

6. J'emploierai les deux termes "ombre" et "double" sans distinction en suivant la définition que Baudouin a empruntée à Jung. ( $B, p .77)$ Je préfére ces termes au "ça” de Freud parce que pour Gabrielle cette partie de sa psyché prend le visage de personnages reels comme la tante Éva, Fœtus, et son père. 
Les mots 'ombre' et 'double' semblent plus ou moins impliquer cette 'personnification'.

7. Dans ce passage, fœetus, symbole du double, est indiqué par "ça ". Cet emploi spécifique du mot ça, qui est très fréquent dans Après la boue, montre qu'en effet le double correspond au «ça» de Freud.

8. $(\mathrm{o})=$ ordinaire; (d) $=$ double; (s) $=$ surmoi. (s)' indique que le surmoi est ici le locuteur.

9. Dorénavant je désignerai ces trois romans par les lettres $N, C$, et $A B$.

10. A savoir le moi authentique. Dans la suite j'employerai dans le même sens les termes: "l'identité», "le vrai moi», "le véritable moi ou comme ici «le moi " tout court. 